\title{
CYTOTOXIC ACTIVITY OF METFORMIN IN VITRO DOES NOT CORRELATE WITH ITS ANTITUMOR ACTION IN VIVO
}

\author{
O.N. Pyaskovskaya, D.L. Kolesnik, A.G. Fedorchuk, G.V. Gorbik, G.I. Solyanik \\ R.E. Kavetsky Institute of Experimental Pathology, Oncology and Radiobiology, \\ NAS of Ukraine, Kyiv 03022, Ukraine
}

It is known that metformin is a hypoglycemic drug used to treat type II diabetes mellitus. Recently active studies of its antitumor activity in relation to different types of malignant cells are conducted. Aim: To determine the relationship between cytotoxic activity of metformin in vitro and its antitumor activity in vivo. Materials and Methods: The rat $\mathrm{C} 6$ glioma cell line and mouse Lewis lung carcinoma cells (LLC) were used in this work. The number of living cells in the cytotoxic test was evaluated using sulforhodamine B. Parameters of tumor cell susceptibility to metformin activity in vitro were calculated using nonlinear and linear regression of experimental data. The antitumor action of metformin in vivo was evaluated routinely by the extension of survival time (ST) (in rats with intracerebral C6 glioma) and its effect on the volume of the primary tumor, the number and volume of metastases (in mice with LLC). Results: In cultured LLC cells in vitro, the proportions of metformin-resistant $\left(A_{1}, \%\right)$ and metformin-sensitive $\left(A_{2}, \%\right)$ subpopulations were $10.0 \pm 2.2 \%$ and $92.0 \pm 3.5 \%$, respectively, in terms of the total number of living cells. Parameter $t$, which characterizes the sensitivity of cancer cells to metformin action (the lower is the value of this parameter the higher is sensitivity of cells to metformin cytotoxicity), for metformin-resistant and metformin-sensitive subpopulations was: $t_{1}(m M)=\infty$ and $t_{2}(m M)=2.9 \pm 0.3$, correspondingly. For metformin-sensitive subpopulation of $L L C$ cells $I_{50}(\mathrm{mM})=\mathbf{2 . 4 2} \pm 0.34$. The volume of the primary tumor, the amount and volume of metastases in mice receiving metformin at a dose of $D_{\min }(0.15 \mathrm{~g} / \mathrm{kg})$ and $D_{\max }(0.3 \mathrm{~g} / \mathrm{kg}) \mathrm{values}$ did not significantly differ from those in the control. However, in the case of $D_{\text {min }}$, there was a tendency to increased volume of the primary tumor, in the case of $D_{\text {max }}$, there was a tendency to increased volume of metastases. The analogical parameters $\left(A_{1}, A_{2}\right.$, $\left.\mathrm{b}_{1}, \mathrm{~b}_{2}, \mathrm{IC}_{50}(1), \mathrm{IC}_{50}(2)\right)$ characterizing cell sensitivity to the action of metformin in vitro were obtained in relation to $\mathrm{C6}$ glioma cells. In metformin-resistant subpopulation, these parameters were: $A_{1}(\%)=72.3 \pm 1.4 ; b_{1}\left(\% / m_{M}\right)=0.43 \pm 0.005 ; I_{50}(1)$ $(\mathrm{mM})=84.1 \pm 2.4$. For metformin-sensitive subpopulation, these parameters were: $A_{2}(\%)=30.8 \pm 2.3 ; b_{2}(\% / m M)=2.87 \pm 0.4 ;$ $\mathrm{IC}_{50}(2)(\mathrm{mM})=5.37 \pm 0.45$. In vivo, a statistically significant anti-glioma effect of metformin was observed: at a dose of $\mathrm{D}_{\max }$ $(5.2 \mathrm{~g} / \mathrm{kg})$ administration of this preparation resulted in a prolongation of the mean ST of tumor-bearing rats by $23 \%(p<0.05)$ compared with that in the control. Conclusions: We found no correlation between the cytotoxic/cytostatic action of metformin in vitro and its antitumor activity in vivo on the two types of tumor cells; these results indicate a significant contribution of the tumor microenvironment to the implementation of the antitumor activity of the drug.

Key Words: C6 glioma, Lewis lung carcinoma, tumor cell sensitivity to metformin.

Presently, metformin, which has long been known as an effective hypoglycemic agent for the treatment of type II diabetes mellitus, is being investigated as a potential antitumor agent. This drug has attracted the attention of oncologists due to clinical observations regarding the reduction in the cancer incidence and cancer-related death rate in diabetic patients treated with metformin [1]. Today, the ability of metformin to suppress the growth of various types of malignant cells has been confirmed in many in vitro and in vivo studies [2-6].

It is believed that the basis of antitumor activity of metformin lies in its ability to moderately inhibit NADH: ubiquinone-oxidoreductase of mitochondria or complex I of the electron transport chain. Complex I plays a key role in the processes of cellular respiration and oxidative phosphorylation and provides almost $40 \%$ of the total proton gradient for the synthesis of ATP in cells [7]. Inhibition of complex I with metformin leads to an increase in the AMP/ADP ratio in the cell and, accordingly, to the activation of the AMP-activated protein kinase (AMPK), main controller

Submitted: October 05, 2017.

*Correspondence: $\quad$ E-mail: pyaskovskaya@gmail.com

Abbreviations used: AMPK - AMP-activated protein kinase; LLC - Lewis lung carcinoma; ST - survival time. of energy metabolism [8-10]. In addition to activating the AMPK pathway, the down regulation of the mTOR signaling, which is critical for the survival and proliferation of tumor cells $[9,11]$, and AMPK-independent mechanisms $[12,13]$ are included in the antitumor pathways affected by metformin. However, the antitumor properties of metformin, persuasive in vitro, are not so rosy in the clinical situation. Although clinical trials of metformin for cancer therapy, in particular breast cancer treatment, are ongoing, there are questions about the effective doses of the drug, the range of tumors that are sensitive to its action, the exact mechanisms of its effects, there is a need for biomarkers of tumor sensitivity to this preparation, etc. [14, 15].

The aim of this work was to determine the relationship between in vitro cytotoxic action of metformin and its antitumor activity in vivo. As an experimental tumor models, we used the Lewis lung carcinoma (LLC) and rat C6 glioma cells.

\section{MATERIALS AND METHODS}

Experimental animals and tumor models. The study was performed in femaleWistar rats $2.5-3$ months old weighting $100-130 \mathrm{~g}$, and C57BL/6 mice 2-2.5 months old weighting $18-22 \mathrm{~g}$ bred at animal facility of R.E. Kavetsky Institute of Experimental Pathology, Oncology and Radiobiology of the National 
Academy of Sciences of Ukraine (IEPOR), Kyiv, Ukraine. The use and care of the experimental animals was performed in accordance with the standard international rules of biologic ethics and was approved by Institutional Animal Care and Use Committee.

Glioma C6 cell line and LLC cells were obtained from the National Bank of Cell Lines and Tumor Strains of IEPOR. Both tumor cells types were maintained in vitro in DMEM (C6 glioma) and RPMI 1640 (LLC) culture medium (Sigma, USA) supplemented with $10 \%$ FBS (Sigma, USA), $2 \mathrm{mM}$ L-glutamine, and $40 \mathrm{mg} / \mathrm{ml}$ gentamycin at $37^{\circ} \mathrm{C}$ in humidified atmosphere with $5 \% \mathrm{CO}_{2}$.

Cytotoxic/cytostatic activity of metformin hydrochloride (Sigma, USA) was assessed by $\mathrm{IC}_{50}$ as the concentration of the agent, which causes a $50 \%$ decrease in the number of living cells in relation to control due to its cytotoxic and/or cytostatic action, which was calculated using an appropriate mathematical model.

For this, C6 glioma cells or LLC cells were placed in wells of 96 -well plates at a density of $1 \cdot 10^{4}$ cells/well, incubated overnight, then the medium was replaced by fresh medium with addition of metformin, and incubation continued for 1 day.

Each concentration of the agent was investigated in repeat. Control cells were incubated under the same conditions without the addition of metformin.

The number of living cells was evaluated using sulforhodamine B (Sigma, USA) [16].

The nature of the dependence of the cytotoxic/ cytostatic action of metformin on LLC cells was analyzed using a mathematical model (1) that takes into account the existence of two subpopulations of tumor cells with varying sensitivity to the action of the drug:

$$
N=A_{1} \cdot \exp \left(-C / t_{1}\right)+A_{2} \cdot \exp \left(-C / t_{2}\right),
$$

where $N$ - number of viable cells (\%), $C$ - metformin concentrations (mM), $A_{1}$ and $A_{2}-$ portion of LLC cells with different sensitivity to metformin action, $t_{1}$ and $t_{2}$ - parameters that characterize the sensitivity of cancer cells to metformin action (the lower the value of this parameter the higher sensitivity of the cells to metformin cytotoxicity).

The relationship between the number of viable C6 glioma cells and metformin concentrations was described by the following mathematical model (taking into account the existence of two subpopulations with different sensitivity to metformin action):

$$
\begin{gathered}
N_{1}=A_{1}-b_{1} \cdot C, \\
N_{2}=A_{2}-b_{2} \cdot C(\text { for } C<9.5 \mathrm{mM}),
\end{gathered}
$$

where $N_{1}$ and $N_{2}$ - the portion of viable cells of the first and second subpopulations; $A_{1}$ and $A_{2}$ - the portion of resistant and sensitive respectively subpopulations in glioma $C 6$ cells; $b_{1}$ and $b_{2}$ - parameters characterizing the sensitivity of cancer cells of each subpopulation to metformin action (the higher the value of this parameter the higher sensitivity of cells to metformin cytotoxicity).

Parameters of mathematical models (1)-(3) were determined from their best approximation to the cor- responding experimental data using nonlinear regression analysis.

Metformin doses and administration mode. Transplantation of LLC cells was performed intramuscularly $\left(1 \cdot 10^{6}\right.$ cells per mouse in $0.1 \mathrm{ml}$ of Hanks' solution).

After transplantation, the mice were randomized by weight and distributed into 3 groups: 1) mice administered with metformin at a total dose of $150 \mathrm{mg} / \mathrm{kg}$ $\left(D_{\min }, \mathrm{n}=8\right)$; 2) mice administered with metformin at a total dose of $300 \mathrm{mg} / \mathrm{kg}\left(D_{\max }, \mathrm{n}=9\right)$ and 3) mice administered with water (control, $n=9$ ) according to the same scheme and in the same volume. Metformin solution was prepared ex tempore on water and administered orally in a volume of $0.5 \mathrm{ml}$, daily, 5 times a week, for three weeks. In total 15 administrations were made.

Transplantation of $\mathrm{C} 6$ glioma cells was performed under total anesthesia via intracerebral inoculation of $0.6 \cdot 10^{6}$ cells per rat in $0.05 \mathrm{ml}$ of physiologic solution in a left parietal area (anterior horn of left lateral ventricle).

After transplantation, the rats were randomized by weight and distributed into 3 groups: rats administered with metformin at a total dose of $2.6 \mathrm{~g} / \mathrm{kg}\left(D_{\min }\right.$, $\mathrm{n}=7$ ); rats administered with metformin at a total dose of $5.2 \mathrm{~g} / \mathrm{kg}\left(D_{\max }, \mathrm{n}=7\right)$, and rats administered with water (control, $n=7$ ) according to the same scheme and in the same volume. Metformin was used at doses close to the most tolerable: the daily dose of the drug was 200 and $400 \mathrm{mg} / \mathrm{kg} /$ day, respectively. According to [17], the first dose in the case of sufficiently long application does not cause significant toxic manifestations, the second one is lower than the dose $(600 \mathrm{mg} / \mathrm{kg} / \mathrm{rat})$, which causes minimal metabolic effects in rats, in particular, increased serum lactate and beta-hydroxybutyric acid and decreased serum bicarbonate and urine $\mathrm{pH}$.

The drug was administered orally in $3 \mathrm{ml}$ of water daily from the day following the tumor transplantation. In total 13 administrations were made.

Evaluation of the effectiveness of the antitumor action of metformin with respect to LLC was performed by the indexes of the effect of the drug on the growth of the primary tumor, the number of pulmonary metastases and their volume in mice after the end of therapy while in $\mathrm{C} 6$ glioma bearing rats - by survival time (ST) of the animals.

The changes in survival time (CST) of rats served as an index of anticancer effect of the metformin calculated by a formula:

$$
\operatorname{CST}(\%)=100 \cdot(S T-S T C) / S T C \text {, }
$$

where $S T$ and STC are survival time of rats from experimental and control groups, respectively.

Evaluation of antitumor and antimetastatic activity of the metformin was performed at day 23 after tumor transplantation. Primary tumor diameter was measured triply per week starting from the moment of development of palpable tumors. Tumor volume (V) was calculated by the formula: 


$$
V=0.52 d^{3}
$$

where $d$ - tumor diameter.

The number and volume of lung metastases was routinely analyzed using binocular microscope and millimeter scale.

Volume of metastases (V) was calculated by the formula:

$$
V=\sum_{1}^{N} \frac{\pi\left(d_{i}\right)^{3}}{n_{i} \cdot 6}
$$

where $n_{i}-$ number of metastases with the diameter of $d_{i}$.

Statistical analysis of the data was performed by descriptive methods, linear and nonlinear regression analysis, Student's t-test with the use of Microsoft Excel and Microcal Origin programs. The data are presented as $\mathrm{M} \pm \mathrm{m}$.

\section{RESULTS AND DISCUSSION}

Effect of metformin on LLC cells in vitro and in vivo. The obtained data on the influence of metformin on the survival of LLC cells in vitro showed a high level of their sensitivity to the action of the drug. Fig. 1 shows the dependence of survival of LLC cells on the concentration of metformin in the incubation medium. As can be seen from Fig. 1, the mathematical model properly described the changes in the number of living cells due to the action of metformin. The determined parameters of the mathematical model for metformin with respect to LLC cells are presented in Table 1.

Table 1. Mathematical model parameters of LLC sensitivity against metformin

\begin{tabular}{ccc}
\hline Symbols & Units & $\begin{array}{c}\text { Value } \\
(\mathrm{M} \pm \mathrm{m})\end{array}$ \\
\hline $\mathrm{A}_{1}$ & $\%$ & $10.0 \pm 2.2$ \\
$\mathrm{~A}_{2}$ & $\%$ & $92.0 \pm 3.5$ \\
$\mathrm{t}_{1}$ & $\mathrm{mM}$ & $\infty$ \\
$\mathrm{t}_{2}$ & $\mathrm{mM}$ & $2.9 \pm 0.3$ \\
$\mathrm{R}^{2}$ & & 0.99 \\
$\mathrm{IC}_{50}$ & $\mathrm{mM}$ & $2.42 \pm 0.34$ \\
\hline
\end{tabular}

The mathematical analysis showed the presence of two subpopulations of LLC cells, which differ significantly in the sensitivity to cytotoxic action of metformin. Subpopulation of cells characterized by high metformin sensitivity (sensitivity index $t_{2}=2.9 \pm 0.3 \mathrm{mM}$ ) achieved more than $90 \%$ of the total number of living cells (Table 1, Fig. 1), causing a sufficiently high overall sensitivity of LLC cells to metformin action, which was confirmed by a low $I C_{50}(2.42 \pm 0.34 \mathrm{mM})$. Despite the fact that the subpopulation of metformin-resistant cells was only about $10 \%$ of cells from the total number of living cells, it is characterized by absolute insensitivity to the action of metformin over a wide range of drug concentrations (parameter $t_{1}=\infty$ ).

Despite the relatively high sensitivity of LLC cells to metformin in vitro, no significant effect of metformin on LLC growth and metastasis was found in vivo. As can be seen from Fig. 2, the volume of primary tumor in mice receiving metformin both at the dose of 150 and $300 \mathrm{mg} / \mathrm{kg}$, did not statistically differ from that in the control animals throughout all period of tumor development.

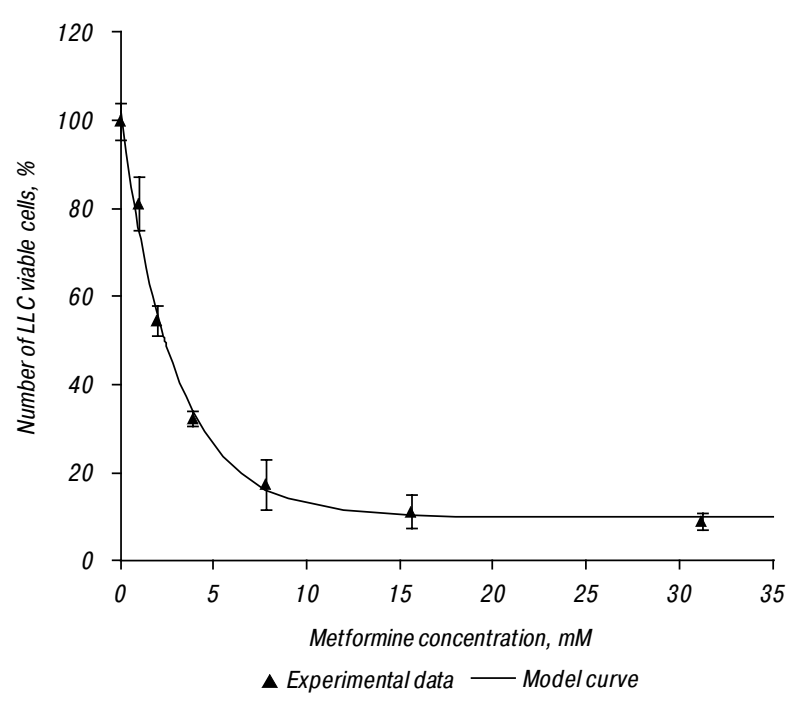

Fig. 1. LLC cell survival as a function of metformin concentration in culture medium. Symbol - experimental data; line model approximation obtained from the best fit of mathematical model (1) to experimental data

At the end of therapy with metformin ( $23^{\text {rd }}$ day after tumor transplantation), no significant effect of the two doses was found regarding both the volume of the primary tumor and metastasis (Table 2). Moreover, the tendency to stimulate the growth of the primary tumor in mice, if metformin was administered at a dose of $150 \mathrm{mg} / \mathrm{kg}$, and a tendency to increase the lung metastasis if mice treated with metformin at a dose of $300 \mathrm{mg} / \mathrm{kg}$ were registered.

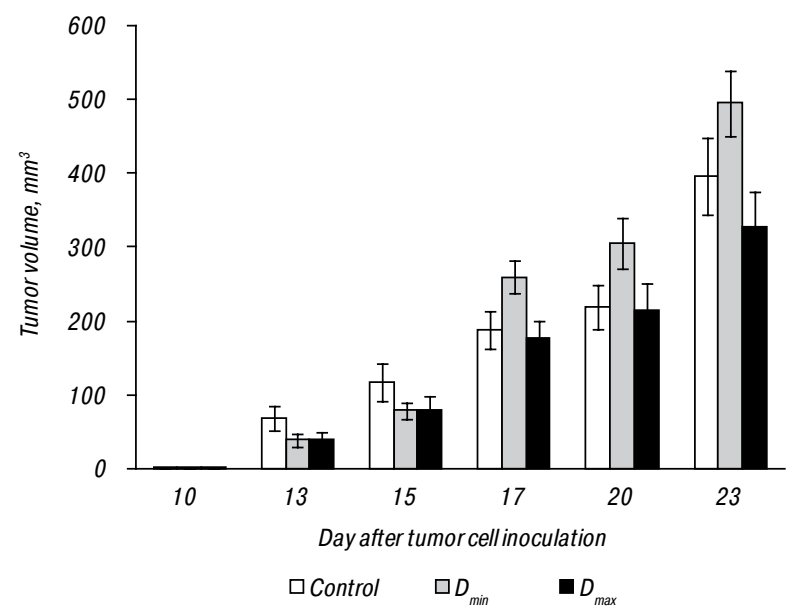

Fig. 2. The effect of metformin on tumor growth in LLC bearing mice

Table 2. The action of metformin on LLC growth and metastasis values

\begin{tabular}{lccc}
\hline \multicolumn{1}{c}{ Group } & $\begin{array}{c}\text { Tumor volume } \\
\left(\mathrm{mm}^{3}\right)\end{array}$ & $\begin{array}{c}\text { Number } \\
\text { of metastases }\end{array}$ & $\begin{array}{c}\text { Volume of metastases } \\
\left(\mathrm{mm}^{3}\right)\end{array}$ \\
\hline Control & $396.0 \pm 52.8$ & $15.9 \pm 4.7$ & $22.3 \pm 7.8$ \\
Metformin $\left(D_{\min }\right)$ & $495.3 \pm 44.1$ & $19.1 \pm 5.2$ & $29.6 \pm 8.5$ \\
Metformin $\left(D_{\max }\right)$ & $327.8 \pm 47.4$ & $21.9 \pm 6.3$ & $53.0 \pm 22.5$ \\
\hline
\end{tabular}

Effect of metformin on $\mathrm{C6}$ glioma cells in vitro and in vivo. The study of the cytotoxic/cytostatic action of metformin in relation to $\mathrm{C} 6$ glioma cells revealed that these cells consist of two subpopulations substantially differing in sensitivity to metformin cytotoxicity (Table 3, Fig. 3). As can be seen from Table 3, cell subpopulation with extremely low sensitivity to metformin action dominated among C6 glioma 
cells. The metformin-resistant subpopulation was about $72 \%$ of all living cells, while metformin-sensitive cells composed only about $28 \%$ of their total number. Accordingly, $\mathrm{IC}_{50}$ for metformin-resistant subpopulation was almost 16 times higher $(p<0.05)$ than that for metformin-sensitive subpopulation.

Table 3. Mathematical model parameters of glioma C6 cell sensitivity against metformin

\begin{tabular}{ccc}
\hline Symbols & Units & $\begin{array}{c}\text { Value } \\
(\mathrm{M} \pm \mathrm{m})\end{array}$ \\
\hline $\mathrm{A}_{1}$ & $\%$ & $72.3 \pm 1.4$ \\
$\mathrm{~b}_{1}$ & $\% / \mathrm{mM}$ & $0.43 \pm 0.005$ \\
$\mathrm{IC}_{50}(1)$ & $\mathrm{mM}$ & $84.1 \pm 2.4$ \\
$\mathrm{~A}_{2}$ & $\%$ & $30.8 \pm 2.3$ \\
$\mathrm{~b}_{2}$ & $\% / \mathrm{mM}$ & $2.87 \pm 0.4$ \\
$\mathrm{IC}_{50}(2)$ & $\mathrm{mM}$ & $5.37 \pm 0.45$ \\
\hline
\end{tabular}

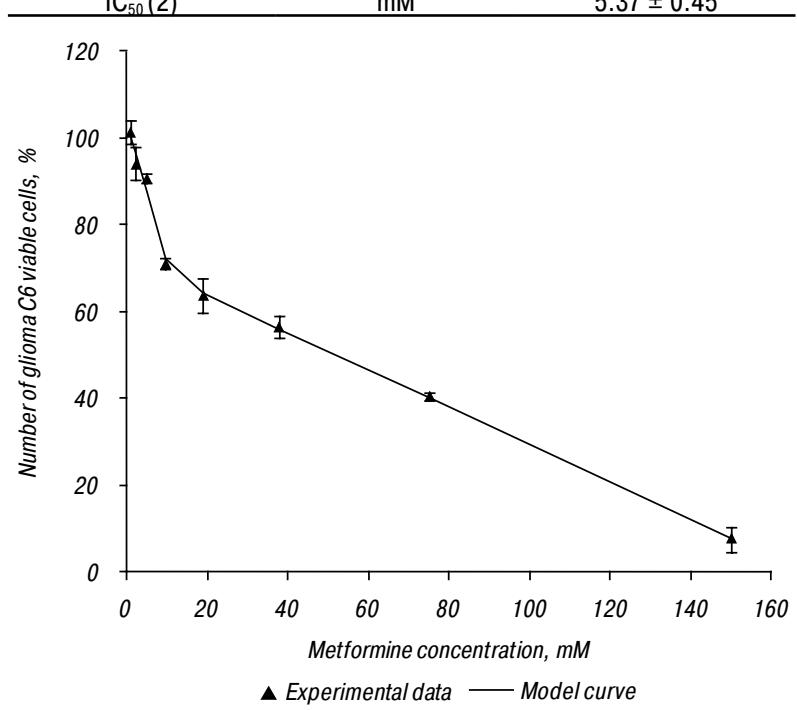

Fig. 3. Glioma cell survival as a function of metformin concentration in culture medium. Symbol - experimental data; line model approximation obtained from the best fit of mathematical model $(2,3)$ to experimental data

Despite the predominance of metformin-resistant glomerular $\mathrm{C} 6$ cells in cell subpopulations in vitro, this drug exhibited a significant anti-glioma effect in in vivo experiments. Anti-glioma action of metformin was confirmed by a significant increase of the ST in rats with transplanted tumors, at least in the maximum dose being studied (Table 4, Fig. 4). In particulars, the average ST of rats treated with metformin at a total dose of $2.6 \mathrm{~g} / \mathrm{kg}$, did not differ significantly from that in the control. Instead, the average ST of rats treated with metformin with twice higher dose, $5.2 \mathrm{~g} / \mathrm{kg}$, was by $23 \%(p<0.05)$ higher than the corresponding index of control animals.

Table 4. The action of metformin on ST of rats with glioma C6

\begin{tabular}{lc}
\hline \multicolumn{1}{c}{ Group } & ST, days \\
\hline Control & 13,1 \\
Metformin $\left(D_{\min }\right)$ & 15,7 \\
Metformin $\left(D_{\max }\right)$ & $16,1^{*}$ \\
\hline
\end{tabular}

Notes: ${ }^{*} p<0.05$ as compared to control.

So, the results of this study showed that, as in the case of LLC, and in the case of C6 glioma, the tumor cell population was heterogeneous and consisted of metformin-resistant and metformin-sensitive subpopulations, and tumor cell susceptibility in vitro did not correlate with their sensitivity to the action of this agent in vivo. In C6 glioma cells cultured in vitro there

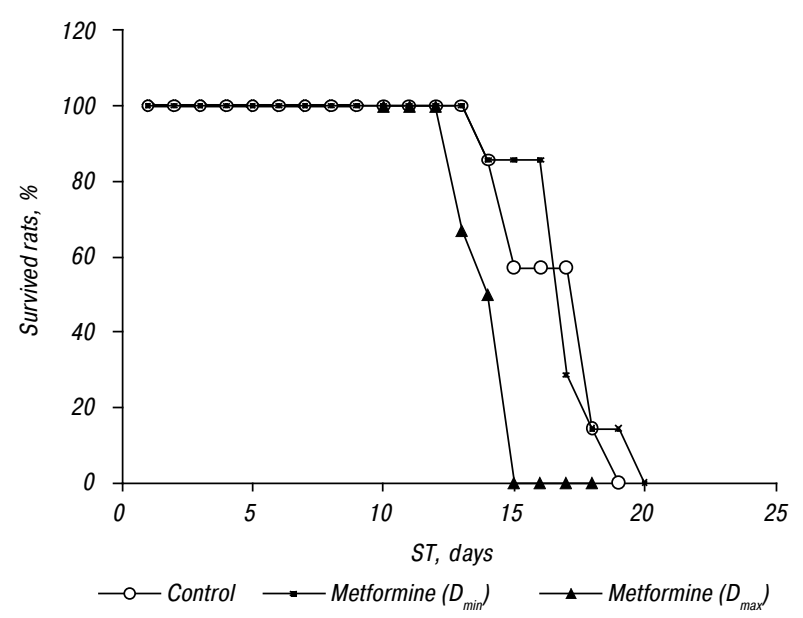

Fig. 4. Survival curves of rats with intracerebral glioma $\mathrm{C} 6$ treated with metformin

was a large predominance of metformin-resistant subpopulation, which by 2.5 times exceeded metformin-sensitive subpopulation. However, despite such dominance of the metformin-resistant cell subpopulation in vitro, metformin exhibited in vivo a pronounced anti-glioma effect, which was manifested by statistically significant prolongation of the lifetime of tumorbearing rats.

In contrast, in the case of LLC, under in vitro conditions, the metformin-sensitive cell subpopulation was almost an order of magnitude larger than such a metformin-resistant subpopulation, which, however, did not lead to the expected antitumor effect of this drug in vivo. Moreover, even a certain trend was observed in stimulating the growth and metastasis of this tumor type.

The revealed effects indicated a lack of correlation between in vitro cytotoxic activity of metformin and its antitumor efficacy in vivo for both types of tumor cells. Such a lack of correlation between the sensitivity of tumor cells to the action of metformin in vitro and in vivo, both in LLC and C6 glioma, indicated a significant contribution of tumor microenvironment to the realization of antitumor action of the drug in vivo.

It is known that the microenvironment of solid tumors is characterized by a shortage of nutrients and hypoxia, which is a consequence of metabolism, first of all, tumor cells. The specific features of tumor microenvironment, undoubtedly, affect the antitumor effectiveness of metformin. Indeed, there is evidence that hypoxia greatly enhances the antiproliferative and apoptotic action of metformin against MCF-7 breast cancer cells [22].

In the case of metformin, the tumor microenvironment is also formed due to its ability to regulate the metabolism of not only tumors, but also many normal cells of the body. In particular, it is known that metformin stimulates the usage of glucose and oxidation of fatty acids by skeletal myocytes, stimulates the oxidation of fatty acids and reduces glucose production in hepatocytes, and, as a result, reduces blood glucose levels $[8,18]$, which results in an even greater fall in glucose content in a tumor microenvironment. 
On this basis, acute glucose deficiency in tumor microenvironment and the sensitivity of tumor cells to such a deficiency may be one of the main causes of inversion of the cytotoxic activity of metformin in relation to LLC and C6 glioma in vitro and in vivo. LLC cells are quite resistant to glucose deficiency due to their ability to pass under these conditions to the G0/G1 phase [19], which is associated with a low antitumor activity of metformin against LLC in vivo. By contrast, $\mathrm{C} 6$ glioma cells possess a high glucose dependence [20], which is clearly associated with a significant anti-glioma effect of this drug. It is also possible that the ability of metformin to reduce the permeability of blood vessels and cerebral edema caused by glioma in rats contributes to its antiglioma action [21].

In conclusion, the data showing the lack of correlation between the in vitro cytotoxic/cytostatic action of metformin and its in vivo antitumor activity indicate an important role of the tumor microenvironment in the realization of its antitumor activity. Given the anti-glycemic mechanism of action of metformin, it is clear that the glucose deficiency itself in the tumor microenvironment and the sensitivity of tumor cells to such a deficiency determine the effectiveness of its antitumor activity against LLC and C6 glioma in vivo.

\section{REFERENCES}

1. Evans JM, Donnelly LA, Emslie-Smith AM, et al. Metformin and reduced risk of cancer in diabetic patients. BMJ 2005; 330: 1304-5.

2. Gotlieb WH, Saumet J, Beauchamp MC, et al. In vitro metformin anti-neoplastic activity in epithelial ovarian cancer. Gynecol Oncol 2008; 110: 246-50.

3. Janjetovic K, Harhaji-Trajkovic L, Misirkic-Marjanovic $\mathbf{M}$, et al. In vitro and in vivo anti-melanoma action of metformin. Eur J Pharmacol 2011; 668: 373-82.

4. Luo Q, Hu D, Hu S, et al. In vitro and in vivo antitumor effect of metformin as a novel therapeutic agent in human oral squamous cell carcinoma. BMC Cancer 2012; 12: 517 (http://www.biomedcentral.com/1471-2407/12/517).

5. Sesen J, Dahan P, Scotland SJ, et al. Metformin ihibits growth of human glioblastoma cells and enhances therapeutic response. PLoS One 2015; 10: e0123721. DOI:10.1371/journal.pone.0123721.

6. Yu Z, Zhao G, Xie G, et al. Metformin and temozolomide act synergistically to inhibit growth of glioma cells and glioma stem cells in vitro and in vivo. Oncotarget 2015; 6: $32930-43$.
7. Efremov RG, Baradaran R, Sazanov LA. The architecture of respiratory complex I. Nature 2010; 465: 441-5.

8. Zhou G, Myers R, Li Y, et al. Role of AMP-activated protein kinase in mechanism of metformin action. J Clin Invest 2001; 108: 1167-74.

9. Zakikhani M, Dowling R, Fantus IG, et al. Metformin is an AMP kinase-dependent growth inhibitor for breast cancer cells. Cancer Res 2006; 66: 10269-73.

10. Owen MR, Doran E, Halestrap AP. Evidence that metformin exerts its anti-diabetic effects through inhibition of complex 1 of the mitochondrial respiratory chain. Biochem J 2000; 348: 607-14.

11. Song CW, Lee H, Dings RP, et al. Metformin kills and radiosensitizes cancer cells and preferentially kills cancer stem cells. Sci Rep 2012; 2: 362. doi: 10.1038/srep00362.

12. Ben Sahra I, Regazzetti C, Robert G, et al. Metformin, independent of AMPK, induces mTOR inhibition and cellcycle arrest through REDD1. Cancer Res 2011; 71: 4366-72.

13. Liu X, Chhipa RR, Pooya S, et al. Discrete mechanisms of mTOR and cell cycle regulation by AMPK agonists independent of AMPK. Proc Natl Acad Sci USA 2014; 111: E435-44.

14. Hatoum D, McGowan EM. Recent advances in the use of metformin: can treating diabetes prevent breast cancer? BioMed Res Int 2015; 2015: Article ID 548436. 13 p. (http://dx.doi.org/10.1155/2015/548436).

15. Camacho L, Dasgupta A, Jiralerspong S. Metformin in breast cancer - an evolving mystery. Breast Cancer Res 2015; 17: 88. DOI 10.1186/s13058-015-0598-8.

16. Vichai V, Kirtikara K. Sulforhodamine B colorimetric assay for cytoxicity screening. Nature Protocol 2006; 1: 1112-6.

17. Quaile MP, Melich DH, Jordan HL, et al. Toxicity and toxicokinetics of metformin in rats. Toxicol Appl Pharmacol 2010; 243: 340-7.

18. Huang NL, Chiang SH, Hsueh CH, et al. Metformin inhibits TNF-alpha-induced IkappaB kinase phosphorylation, IkappaB-alpha degradation and IL-6 production in endothelial cells through PI3K-dependent AMPK phosphorylation. Int J Cardiol 2009; 134: 169-75.

19. Pyaskovskaya ON, Kolesnik DL, Kolobov AV, et al. Analysis of growth kinetics and proliferative heterogeneity of Lewis lung carcinoma cells growing as unfed culture. Exp Oncol 2008; 30: 269-75.

20. Seyfried TN, Sanderson TM, El-Abbadi MM, et al. Role of glucose and ketone bodies in the metabolic control of experimental brain cancer. Br J Cancer 2003; 89: 1375-82.

21. Zhao B, Wang X, Zheng J et al. Effects of metformin treatment on glioma-induced brain edema. Am J Transl Res 2016; 8: 3351-63.

22. Safari Z, Safaralizadeh R, Seyedzadeh MH, et al. The Induction of metformin inhibitory effects on tumor cell growth in hypoxic condition. Iran J Allergy Asthma Immunol 2015; 14: 605-14. 